ARTICLE

Received 21 Mar 2016 | Accepted 7 Jun 2016 | Published 20 Jul 2016 | Updated 31 Aug 2016

DOI: $10.1038 /$ ncomms12195

\title{
Iridates from the molecular side
}

\author{
Kasper S. Pedersen 1,2,3,4, Jesper Bendix ${ }^{5}$, Alain Tressaud ${ }^{3,4}$, Etienne Durand ${ }^{3,4}$, Høgni Weihe ${ }^{5}$, Zaher Salman6, \\ Thorbjørn J. Morsing 5 , Daniel N. Woodruff7, Yanhua Lan ${ }^{8}$, Wolfgang Wernsdorfer ${ }^{8}$, Corine Mathonière ${ }^{3,4}$, \\ Stergios Piligkos ${ }^{5}$, Sophia I. Klokishner ${ }^{9}$, Serghei Ostrovsky ${ }^{9}$, Katharina Ollefs ${ }^{10}{ }^{\dagger}$, Fabrice Wilhelm $^{10}$, \\ Andrei Rogalev ${ }^{10} \&$ Rodolphe Clérac ${ }^{1,2}$
}

New exotic phenomena have recently been discovered in oxides of paramagnetic $\mathrm{Ir}^{4+}$ ions, widely known as 'iridates'. Their remarkable properties originate from concerted effects of the crystal field, magnetic interactions and strong spin-orbit coupling, characteristic of $5 \mathrm{~d}$ metal ions. Despite numerous experimental reports, the electronic structure of these materials is still challenging to elucidate, and not attainable in the isolated, but chemically inaccessible, $\left[\mathrm{IrO}_{6}\right]^{8-}$ species (the simplest molecular analogue of the elementary $\left\{\mathrm{IrO}_{6}\right\}^{8-}$ fragment present in all iridates). Here, we introduce an alternative approach to circumvent this problem by substituting the oxide ions in $\left[\mathrm{IrO}_{6}\right]^{8-}$ by isoelectronic fluorides to form the fluorido-iridate: $\left[\mathrm{lrF}_{6}\right]^{2-}$. This molecular species has the same electronic ground state as the $\left\{\mid \mathrm{rO}_{6}\right\}^{8-}$ fragment, and thus emerges as an ideal model for iridates. These results may open perspectives for using fluorido-iridates as building-blocks for electronic and magnetic quantum materials synthesized by soft chemistry routes.

\footnotetext{
${ }^{1}$ CNRS, CRPP, UPR 8641, Pessac 33600, France. ${ }^{2}$ Univ. Bordeaux, CRPP, UPR 8641, Pessac 33600, France. ${ }^{3}$ CNRS, ICMCB, UPR 9048, Pessac 33600 , France. ${ }^{4}$ Univ. Bordeaux, ICMCB, UPR 9048, Pessac 33600, France. ${ }^{5}$ Department of Chemistry, University of Copenhagen, Copenhagen DK-2100, Denmark. ${ }^{6}$ Laboratory for Muon Spin Spectroscopy, Paul Scherrer Institut, Villigen PSI CH-5232, Switzerland. ${ }^{7}$ Department of Chemistry, University of Oxford, Oxford OX1 3QR, UK. ${ }^{8}$ CNRS, Inst NEEL, Grenoble F-38000, France. ${ }^{9}$ Institute of Applied Physics, Academy of Sciences of Moldova, Kishinev 2028, Moldova. 10 ESRF - The European Synchrotron, CS 40220, 38043 Grenoble Cedex 9, France. †Present address: Faculty of Physics and Center for Nanointegration Duisburg-Essen (CENIDE), Universität Duisburg-Essen, 47048 Duisburg, Germany. Correspondence and requests for materials should be addressed to K.S.P. (email: pedersen@crpp-bordeaux.cnrs.fr) or to J.B. (email: bendix@kiku.dk) or to R.C. (email: clerac@crpp-bordeaux.cnrs.fr).
} 
$\mathrm{T}$ he $5 \mathrm{~d}$ elements of the periodic table possess singular properties including a strong coupling of the electronic spin to its orbit as well as spatially extended valence orbitals leading to a reduced electronic repulsion and large effect on the ligand field. These intrinsic characteristics have been experimentally or theoretically demonstrated to be responsible for new exotic states of matter such as spin-orbit (SO) Mott insulators ${ }^{1,2}$, topological insulators ${ }^{3-5}$, super-conductors ${ }^{6-8}$, spin-liquids and -ices ${ }^{9-11}$ and for quantum metal-insulator transitions ${ }^{12}$. The common denominator in these promising materials, collectively referred to as 'iridates' (herein named oxido-iridates), is the presence of octahedrally coordinated $\mathrm{Ir}^{\mathrm{IV}}$ ions (Fig. 1a,b) featuring a $t_{2 g}^{5}$ electronic configuration. The half-filled $j_{\text {eff }}=1 / 2$ level (Fig. 1c) ${ }^{13}$, resulting from the SO coupling, is at the origin of a narrow band gap responsible for the abovementioned phenomena. In all oxido-iridates including $\mathrm{Sr}_{2} \mathrm{IrO}_{4}$, the archetypal SO Mott insulator ${ }^{2}$, corner- or edgesharing $\left\{\mathrm{IrO}_{6}\right\}^{8-}$ distorted octahedra are present (Fig. 1a). While magnetic $\mathrm{Ir}^{\mathrm{IV}}-\mathrm{Ir}^{\mathrm{IV}}$ interactions and their implications for the physical properties have been studied extensively in oxidoiridates ${ }^{14,15}$, the intrinsic magnetic properties ${ }^{16}$ of the elemental $\left\{\mathrm{IrO}_{6}\right\}^{8-}$ moiety are consistently masked by the same long-range interactions. Birol and Haule ${ }^{17}$ recently suggested that the design of materials incorporating isolated $\mathrm{Ir}^{\mathrm{IV}}$ octahedra should facilitate smaller bandwidths and promote Mott ground states. If this strategy is developed to its logical end, a discouraging result is obtained as the isolation of the $\left[\mathrm{IrO}_{6}\right]^{8-}$ ion (Fig. 1b) is chemically impossible. However for the realization of dimensionally reduced oxido-iridates, the fluoride ion appears as an ideal substitute to oxide being isoelectronic with comparable chemical and physical characteristics, but, importantly, with a reduced charge. Along this idea, we present the synthesis of molecular fluorido-iridates incorporating spatially isolated $\left[\mathrm{IrF}_{6}\right]^{2-}$ units. Their local magnetic properties are probed by $\mathrm{X}$-ray magnetic circular dichroism (XMCD) spectroscopy demonstrating that the $\left[\mathrm{IrF}_{6}\right]^{2-}$ and $\left\{\mathrm{IrO}_{6}\right\}^{8-}$ units possess virtually identical electronic ground states as suggested by theory ${ }^{17}$. Thus this fluorido-iridate moiety and its intrinsic properties, experimentally determined in this work, can be confidently used to model and emulate the basic $\left\{\mathrm{IrO}_{6}\right\}^{8-}$ unit in oxido-iridates.

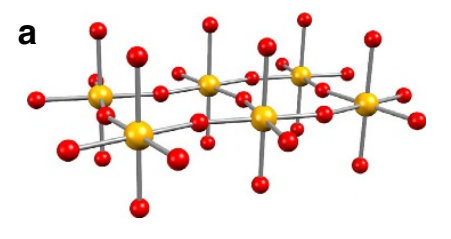

b
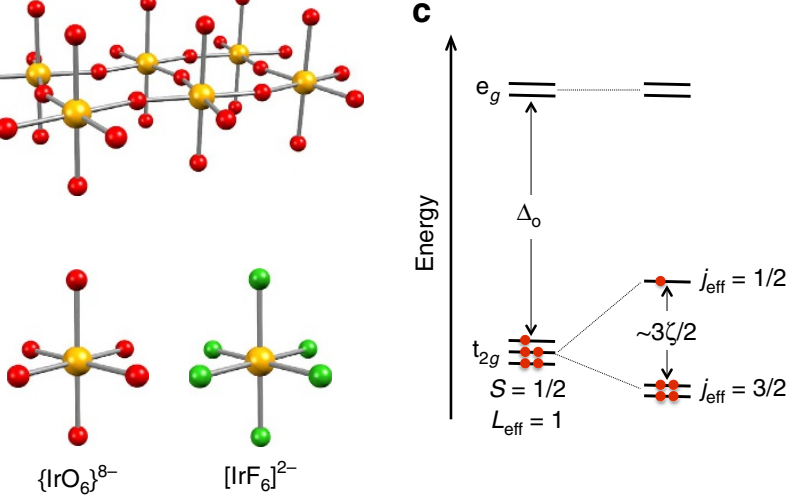

Figure 1 | Oxido-iridates versus fluorido-iridates. Ball and stick

representations of (a) the oxido $\mathrm{Ir}^{\mathrm{IV}}$-based layer in $\mathrm{Sr}_{2} \mathrm{IrO}_{4}$, (b) its smallest $\left\{\mathrm{IrO}_{6}\right\}^{8-}$ unit and the isoelectronic fluoride counterpart, $\left[\mathrm{IrF}_{6}\right]^{2-}$.

(c) Energy level diagram for $\mathrm{Ir}^{\mathrm{IV}}$ ( $5 \mathrm{~d}^{5} ; \mathrm{t}_{2 g}{ }^{5}$ electronic configuration). The octahedral ligand field splits the $5 \mathrm{~d}$ orbitals into $\mathrm{e}_{g}$ and $\mathrm{t}_{2 g}$ levels, and SO coupling further lifts the degeneracy of the $t_{2 g}$ levels into filled $j_{\text {eff }}=3 / 2$ and half-filled $j_{\text {eff }}=1 / 2$ levels. Note that this one-electron picture is equivalent to the SO splitting of the ${ }^{2} \mathrm{~T}_{2 g}\left(\mathrm{O}_{\mathrm{h}}\right)$ term into an $J_{\text {eff }}=1 / 2$ ground state and excited $J_{\text {eff }}=3 / 2$ state.

\section{Results}

Syntheses and structures. Highly water-soluble $\mathrm{Na}_{2}\left[\mathrm{IrF}_{6}\right]$ was obtained by direct $\mathrm{F}_{2}$ fluorination of $\mathrm{Na}_{2}\left[\mathrm{IrCl}_{6}\right] \cdot 6 \mathrm{H}_{2} \mathrm{O}$ at elevated temperature $\left(200^{\circ} \mathrm{C}\right)$ and characterized by powder X-ray diffraction ${ }^{18-20}$. Addition of a solution of $\mathrm{PPh}_{4} \mathrm{Cl}$ $\left(\mathrm{PPh}_{4}^{+}=\right.$tetraphenylphosphonium $(\mathrm{V})$ cation) to an aqueous $\left[\mathrm{IrF}_{6}\right]^{2-}$ solution yielded $\left(\mathrm{PPh}_{4}\right)_{2}\left[\operatorname{IrF}_{6}\right] \cdot 2 \mathrm{H}_{2} \mathrm{O}(1$, Fig. $2 \mathrm{a}$ and Supplementary Fig. 1). Electrospray mass spectrometry on 1 indicated complete absence of any hydrolysis products such as $\left[\operatorname{IrF}_{5}(\mathrm{OH})\right]^{2-}$ (Supplementary Fig. 2). 1 was subsequently used to synthesize $\mathrm{Zn}\left(\right.$ viz $_{4}\left[\mathrm{IrF}_{6}\right]$ (2, Fig. 2b and Supplementary Fig. 3; $\mathrm{viz}=1$-vinylimidazole). These compounds incorporate a low symmetry, but close-to-octahedral $\left[\operatorname{IrF}_{6}\right]^{2-}$ unit in $\mathbf{1}(P \overline{1}$ space group) and a tetragonally distorted octahedral $\left[\mathrm{IrF}_{6}\right]^{2-}$ unit in 2 $\left(P 4_{2} / n\right.$ space group). The axial elongation in both $\mathbf{1}$ and $\mathbf{2}$ is $\sim 1 \%$, slightly smaller than, for example, the $\sim 3 \%$ found in $\mathrm{Sr}_{2} \mathrm{IrO}_{4}$, and the bond angles are all within 1.5 and $0.1 \%$ of $90^{\circ}$, respectively. To elucidate any differences in the $\operatorname{Ir}^{\mathrm{IV}}$ electronic structure when modifying the ligand field, we included in this study the related chloride complex, $\left(\mathrm{PPh}_{4}\right)_{2}\left[\mathrm{IrCl}_{6}\right]$ (3, Fig. $2 \mathrm{c}$ and Supplementary Fig. 4), also featuring an approximately octahedral, but axially compressed $(\sim 0.7 \%)\left[\mathrm{IrCl}_{6}\right]^{2-}$ ion. The nearest neighbour Ir-Ir distances $(\AA)$ are $10.0(1), 8.1(2)$ and 10.1 (3), which are much longer than in the oxido-iridates (for example, $3.9 \AA$ in $\left.\mathrm{Sr}_{2} \mathrm{IrO}_{4}\right)$. Thus the $\mathrm{Ir}^{\mathrm{IV}}-\mathrm{Ir}^{\mathrm{IV}}$ interactions are negligibly small and the $\left[\operatorname{IrX}_{6}\right]^{2-}(\mathrm{X}=\mathrm{F}, \mathrm{Cl})$ unit can be considered as magnetically isolated in 1, 2 and $\mathbf{3}$.

Magnetic properties and X-ray spectroscopy. The susceptibilitytemperature product, $\chi T$, of $\mathbf{1 - 3}$ (Supplementary Fig. 5) is practically temperature independent (Curie's law) confirming the lack of significant magnetic interactions between $\operatorname{Ir}^{\mathrm{IV}}$ spins and thus the absence of any magnetic order at least down to $1.8 \mathrm{~K}$. The complete set of magnetization data ( $M$ versus $\mu_{0} H T^{-1}$; Supplementary Figs 6-8) could be fitted to the Brillouin function for an effective spin-1/2 (with $g \approx 2$ ), which corroborates a

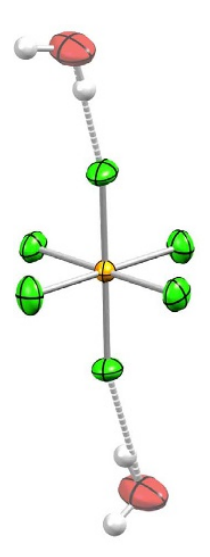

b

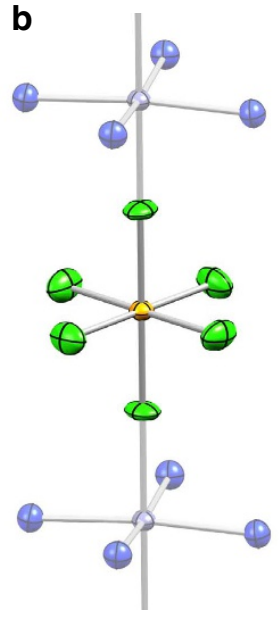

c

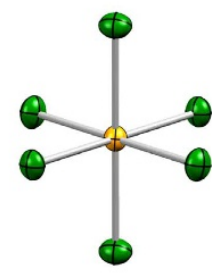

Figure 2 | X-ray structure view of the molecular iridates. Thermal ellipsoid plots of $\mathbf{1}$ (a), $\mathbf{2}$ (b) and $\mathbf{3}$ (c) are shown at $80 \%$ probability level. The counterions for $\mathbf{1}$ and $\mathbf{3}$ and the auxiliary parts of the 1-vinylimidazole ligands in $\mathbf{2}$ are omitted for clarity. Colour code: Ir, yellow; $\mathrm{Zn}$, grey; $\mathrm{Cl}$, dark green; $\mathrm{F}$, pale green; $\mathrm{O}$, red; $\mathrm{N}$, blue; $\mathrm{H}$, white. Selected bond lengths $(\AA)$ and angles $\left(^{\circ}\right)$ for $\mathbf{1}$ : Ir-F 1.9339(8)-1.9510(8), F-Ir- $F_{\text {cis }} 88.89(4)-91.35(4)$; for $\mathbf{2}$ : Ir-F 1.9281(1), 1.9449(1) (only two crystallographically different bond lengths), $\mathrm{F}-\mathrm{Ir}-\mathrm{F}_{\text {cis }}$ 90, 90.0112(1); for 3: Ir-Cl 2.3205(3)-2.3370(3), $\mathrm{Cl}-\mathrm{Ir}-\mathrm{Cl}_{\text {cis }} 88.54(1)-91.47(1)$. 
the Curie susceptibility and demonstrates the presence of an energetically isolated $J_{\text {eff }}=1 / 2$ ground state.

Orbital $\left(M_{\text {orbital }}\right)$ and spin $\left(M_{\text {spin }}\right)$ contributions to the total magnetic moments $\left(M_{\text {total }}\right)$ have been determined experimentally using XMCD, which is defined as the difference between two X-ray absorption spectra (XAS) recorded with either opposite helicity or magnetization direction. XAS spectra were collected on 1-3 at the iridium $L_{2,3}$ edges under an external magnetic field of $\mu_{0} H= \pm 17 \mathrm{~T}$ and at low temperatures $(T=2.6-2.9 \mathrm{~K})$. Since the measurements were performed on powdered samples, the isotropic $L_{2,3}$ XAS spectra could be approximated as $\left(\sigma^{+}+\sigma^{-}\right) / 2$, where $\sigma^{+}\left(\sigma^{-}\right)$is the absorption cross-section obtained with helicity and magnetization aligned either parallel $(+)$ or antiparallel $(-)$ (Fig. 3). The XAS spectra are dominated by strong resonance peaks ('white lines') at both the $L_{3}$ $\left(2 \mathrm{p}_{3 / 2} \rightarrow 5 \mathrm{~d}_{3 / 2,5 / 2}\right)$ and $L_{2}\left(2 \mathrm{p}_{1 / 2} \rightarrow 5 \mathrm{~d}_{3 / 2}\right)$ edges with the $L_{3}$ edge being significantly more intense.

The spectra of $\mathbf{1}$ and $\mathbf{2}$ are virtually identical, whereas for $\mathbf{3}$, a slight shift of the white line peak of $c a .2 \mathrm{eV}$ towards lower energies is observed concomitantly with an additional component at ca. $12 \mathrm{eV}$ higher energy (Supplementary Fig. 9). The latter feature is likely the signature of excitations into delocalized states

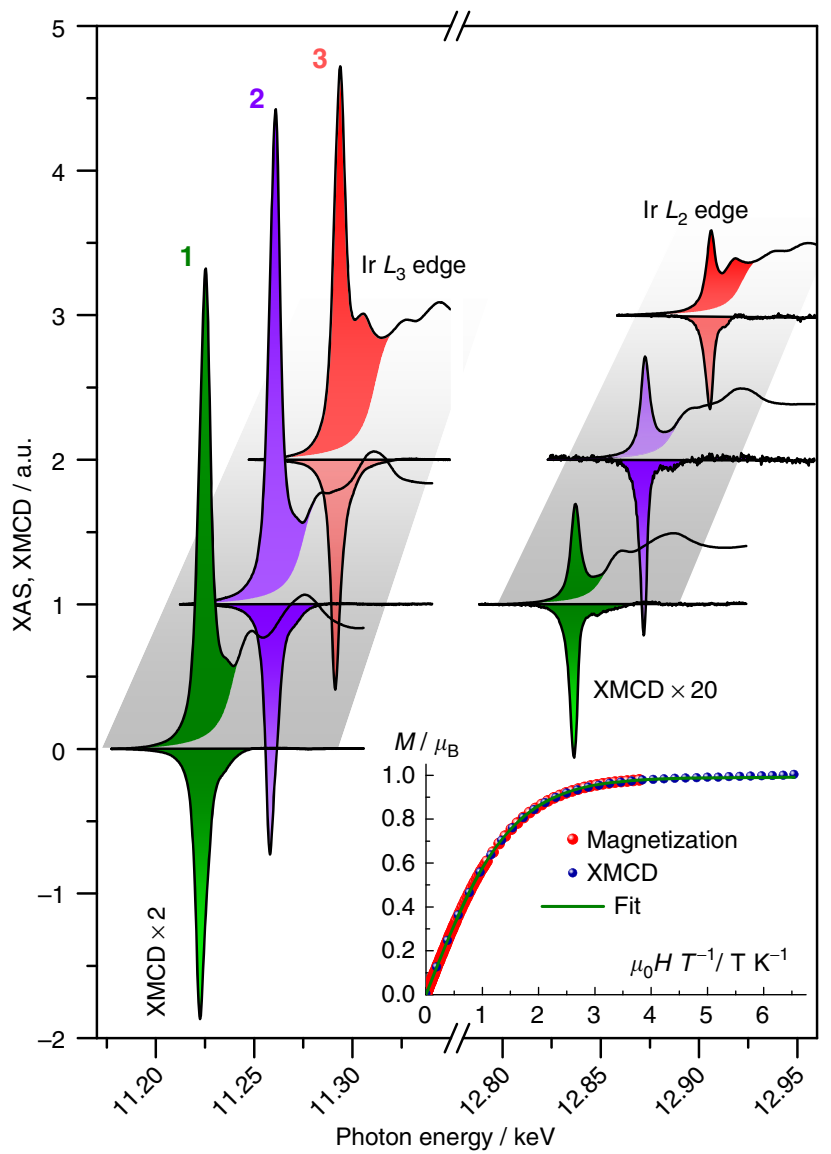

Figure 3 | X-ray spectroscopy. X-ray spectra of 1-3 showing the isotropic XAS (positive values) and XMCD (negative values) obtained in a magnetic field of $+17 \mathrm{~T}$ (at 2.7, 2.6 and $2.5 \mathrm{~K}$ for $\mathbf{1}, \mathbf{2}$ and $\mathbf{3}$ respectively). The spectra of $\mathbf{2}$ and $\mathbf{3}$ were shifted horizontally ( 35 and $70 \mathrm{eV}$, respectively) and vertically for clarity. The filled patterns are the integrals used for the sum rule analysis. Inset: Field dependence of the magnetization, $M$ versus $\mu_{0} H T^{-1}$, of a polycrystalline sample of 2 at $T=2.0 \mathrm{~K}$. 'XMCD' designates the field dependence of the XMCD maximum signal at the $\operatorname{Ir} L_{3}$ edge at $T=2.6 \mathrm{~K}$. The green line is the best fit of the magnetization and scaled XMCD data to the Brillouin function. originating from ligand to metal charge transfer that is not observed in $\mathbf{1}$ and $\mathbf{2}$ due to the more ionic $\mathrm{Ir}-\mathrm{F}$ bond. The effect of SO coupling on the $5 \mathrm{~d}$ states was quantified through the so-called $\mathrm{SO}$ sum rule $\mathrm{e}^{21}$. It relates the branching ratio (BR) of the white line integrals at the $\mathrm{SO}$ split absorption edges to the expectation value of the angular part of the ground state SO operator per hole. For the $\mathrm{p} \rightarrow \mathrm{d}$ transitions ( $L_{2}$ and $L_{3}$ edges), the branching ratio can be expressed as

$$
\mathrm{BR}=\frac{I_{L_{3}}^{\mathrm{XAS}}}{I_{L_{3}}^{\mathrm{XAS}}+I_{L_{2}}^{\mathrm{XAS}}}=\frac{2}{3}-\frac{\left\langle\sum_{i} \mathbf{l}_{i} \cdot \mathbf{s}_{i}\right\rangle}{3\left\langle n_{\mathrm{h}}\right\rangle}
$$

where, $\left\langle n_{\mathrm{h}}\right\rangle=\left\langle n_{\mathrm{h}}{ }^{j=3 / 2}\right\rangle+\left\langle n_{\mathrm{h}}{ }^{j=5 / 2}\right\rangle$ is the total number of holes in the $5 \mathrm{~d}$ levels and $\left\langle\sum_{i} \mathbf{l}_{i} \cdot \mathbf{s}_{i}\right\rangle / \hbar^{2}=-3 / 2 \times\left\langle n_{\mathrm{e}}^{j=3 / 2}\right\rangle+\left\langle n_{\mathrm{e}}^{j=5 / 2}\right\rangle$ is the expectation value of the one-electron $\mathbf{l}_{i} \cdot \mathbf{s}_{i}$ operator summed over all electrons; $\left\langle n_{\mathrm{e}}\right\rangle$ being the occupation number of the corresponding levels. The white line integrals are larger in $\mathbf{3}$ than in 1 or 2 , thus more holes in the $5 \mathrm{~d}$ band should be present for 3 compared with $\mathbf{1}$ or $\mathbf{2}$. If one assumes that $\mathbf{1}$ and $\mathbf{2}$ are completely ionic, $\left\langle n_{\mathrm{h}}\right\rangle=5$, we obtain $\left\langle n_{\mathrm{h}}\right\rangle=5.26$ for 3 . The corresponding results are shown in Table 1 . The quantitative analysis of the XMCD spectra was performed by means of the magneto-optical sum rules providing direct access to $M_{\text {orbital }}=-\left\langle L_{z}\right\rangle \mu_{\mathrm{B}}$ and $M_{\text {spin,eff }}=-2\left\langle S_{\text {eff }}\right\rangle \mu_{\mathrm{B}}$ (the details of this analysis are given in the 'Methods' section; Table 1$)^{22,23}$. The scaling of the field dependence of the XMCD to the bulk magnetization (inset Fig. 3 and Supplementary Fig. 10; see the 'Methods' sections) allows the determination of the absolute value of the magnetization at $\mu_{0} H=+17 \mathrm{~T}: M_{\text {total }}=1.03,1.0$ and $0.96 \mu_{\mathrm{B}}$ for 1, 2 and 3 respectively. From these values, the magnetic dipole contribution, $\left\langle T_{z}\right\rangle$, and $M_{\text {spin }}$ (given by $M_{\text {spin,eff }}+7\left\langle T_{z}\right\rangle$ ) can be estimated (Table 1) without the need of any sophisticated theoretical modelling from $M_{\text {total }}=M_{\text {spin }}+M_{\text {orbital }}$ considering $M_{\text {spin,eff }}$ and $M_{\text {orbital }}$ deduced from the sum rules. The magnetic dipole contribution has never been experimentally determined for any iridate systems, but according to these results, $\left\langle T_{z}\right\rangle$ cannot be neglected without a significant underestimation of the $M_{\text {orbital }} /$ $M_{\text {spin }}$ ratio, as previously anticipated ${ }^{24}$.

Theoretical considerations. The molecular nature of 1, 2 and 3 implies that intuitive localized bonding models should be able to disentangle the magnetic moment contributions. The angular overlap model (AOM) allows for a decomposition of the ligand field potential into $\sigma$ - and $\pi$-bonding parameters ${ }^{25}$. As a first approximation, a cubic $\left[\mathrm{IrF}_{6}\right]^{2-}$ model considering only $\sigma$-bonding (with $\Delta_{O}=27,000 \mathrm{~cm}^{-1}$, Racah interelectronic repulsion parameters $B=510 \mathrm{~cm}^{-1}$ and $C / B=4.9$, and the SO coupling constant $\zeta=3,300 \mathrm{~cm}^{-1}$; Fig. 1c) ${ }^{26}$ affords $\left\langle\sum_{i} \mathbf{1}_{i} \cdot \mathbf{s}_{i}\right\rangle / \hbar^{2}=-2.65$, $M_{\text {orbital }}=0.74 \mu_{\mathrm{B}}$ and $M_{\text {spin }}=0.36 \mu_{\mathrm{B}}$, which are close to the ionic values $\left(M_{\text {orbital }}=0.67 \mu_{\mathrm{B}}\right.$ and $\left.M_{\text {spin }}=0.33 \mu_{\mathrm{B}}\right)$ for a pure $J_{\text {eff }}=1 / 2$ system $^{2}$. Whereas the agreement with the experimental results of the fluorido-iridates (Table 1) for $\left\langle\sum_{i} \mathbf{l}_{i} \cdot \mathbf{s}_{i}\right\rangle / \hbar^{2}$ and $M_{\text {orbital }}$ is

Table 1 | Summary of the $\mathrm{X}$-ray spectroscopy results.

\begin{tabular}{lccc} 
& $\mathbf{1}$ & $\mathbf{2}$ & $\mathbf{3}$ \\
\hline Branching ratio & 0.85 & 0.85 & 0.82 \\
$\left\langle\sum_{i} \mathbf{I}_{i} \cdot \mathbf{s}_{i}\right\rangle\left(\hbar^{2}\right)$ & -2.8 & -2.7 & -2.5 \\
$\left\langle n_{\mathrm{h}}^{j=5}\right\rangle /\left\langle n_{\mathrm{h}}{ }^{j}=3 / 2\right\rangle$ & 4.7 & 4.4 & 3.8 \\
$M_{\text {total }}\left(\mu_{\mathrm{B}}\right)$ & 1.03 & 1.00 & 0.96 \\
$M_{\text {spin }}\left(\mu_{\mathrm{B}}\right)$ & 0.24 & 0.23 & 0.31 \\
$M_{\text {orbital }}\left(\mu_{\mathrm{B}}\right)$ & 0.79 & 0.77 & 0.65 \\
$\left\langle T_{z}\right\rangle\left(\mu_{\mathrm{B}}\right)$ & -0.11 & -0.10 & -0.081 \\
\hline Obtained with a magnetic field of $\mu_{0} \mathrm{H}=17 \mathrm{~T}$. & & \\
\hline
\end{tabular}


surprisingly good, $M_{\text {spin }}$ is overestimated in this simplistic approach which neglects the covalency. A more realistic model including $\pi$-bonding and scaled AOM parameters to account for the different Ir-F bond lengths (see the 'Methods' section) leads to the same conclusion: $\left\langle\sum_{i} \mathbf{l}_{i} \cdot \mathbf{s}_{i}\right\rangle / \hbar^{2}=-2.65, M_{\text {orbital }}=0.72 \mu_{\mathrm{B}}$ and $M_{\text {spin }}=0.32 \mu_{\mathrm{B}}$. However, when covalency is explicitly accounted for by CASSCF calculations, the spin contribution $\left(M_{\text {spin }}=0.22 \mu_{\mathrm{B}}\right)$ is in much better agreement with the experiment, but the orbital contribution becomes slightly underestimated $\left(M_{\text {orbital }}=0.53 \mu_{\mathrm{B}}\right)$. Indeed, this departure from cubic symmetry has little influence on the energy of the first excited $J_{\text {eff }}=3 / 2$ state (see Fig. 1 caption) as evidenced by calculations (Supplementary Table 2) and near-IR absorption spectroscopy revealing absorption bands from $\sim 6,000$ to $\sim 8,500 \mathrm{~cm}^{-1}$ (Supplementary Fig. 11).

Electron paramagnetic resonance spectroscopy. For an ideal $J_{\text {eff }}=1 / 2$ state, magnetic anisotropy is absent, but minuscule deviations from cubic symmetry may result in strong $g$-factor anisotropy that can be probed experimentally by electron paramagnetic resonance (EPR) spectroscopy. The X-band $(v=9.634$ $\mathrm{GHz})$ EPR spectrum of an $\left[\mathrm{IrF}_{6}\right]^{2-}$ doped $\mathrm{Zn}(\mathrm{viz})_{4}\left[\mathrm{ZrF}_{6}\right]$ single crystal ( $\sim 1 \%$ Ir) was measured at $5 \mathrm{~K}$ (Supplementary Figs 12-16; Table 2). The experimental eigenvalues of the $g$-tensor are indeed remarkably anisotropic with $g_{z}=1.37$ and $g_{x y}=2.11$ in good agreement with the CASSCF calculations leading to $g_{z}=1.30$ and $g_{x y}=2.24$ (Supplementary Table 2).

These combined experimental and theoretical results establish that the electronic ground state of the molecular $\left[\mathrm{IrF}_{6}\right]^{2-}$ species is $J_{\text {eff }}=1 / 2$ as suggested for the $\left\{\mathrm{IrO}_{6}\right\}^{8-}$ unit, present in all oxido-iridates.

Dynamic magnetic properties. The magnetization dynamics of the molecular fluorido-iridates was studied by a.c. susceptibility of 2 at different temperatures below $20 \mathrm{~K}$ (Fig. 4 and Supplementary Figs. 17-22 for 1, 2 and 3). The presence of peaks in the a.c. frequency $(v)$ dependence of the imaginary component, $\chi^{\prime \prime} T$ (Fig. 4b), which shift with temperature, clearly indicates the slow relaxation of the magnetization, while the vanishing of the real component, $\chi^{\prime} T$, in the adiabatic limit $(\nu \rightarrow \infty)$ reveals a blocking of the magnetization that concerns the whole volume of the material. Note that for the three compounds, the slow dynamics of the magnetization is observed only on the application of a small static magnetic field that likely serves to decouple the $\operatorname{Ir}^{\mathrm{IV}}$ $J_{\text {eff }}=1 / 2$ from nuclear spins as justified by a comparable magnitude of the applied field $(75 \mathrm{mT})$ to the width of the EPR spectra (Supplementary Fig. 15).

The real and imaginary components of the a.c. susceptibility could be well-fitted to a generalized Debye model ${ }^{27}$ with small values of the distribution parameter (Supplementary Fig. 23) reflecting a single characteristic relaxation time. The temperature dependence of the extracted relaxation time is shown for 2 in Fig. 4 (and also Supplementary Fig. 24 for 1-3). In these molecular iridates, the spin-lattice relaxation rates $\tau^{-1}$ were modelled considering Raman and phonon-bottlenecked direct processes, described as a sum of power laws, $\tau^{-1}=C T^{7}+D T^{2}$, and leading to $C=46 \times 10^{-3} \mathrm{~s}^{-1} \mathrm{~K}^{-7}, D=17 \mathrm{~s}^{-1} \mathrm{~K}^{-2}$ for 2 (ref. 28). The magnetization dynamics appears to be similar in 1, 2 and 3 (Supplementary Fig. 24), suggesting that this property is an intrinsic characteristic of the $\mathrm{Ir}^{\mathrm{IV}}$ electronic structure. The slow relaxation of the magnetization in $\mathbf{2}$ was confirmed by muon spin relaxation $\left(\mu^{+} \mathrm{SR}\right)$ measurements at low temperatures (above $1.9 \mathrm{~K}$; Supplementary Figs 25 and 26), where the implanted muons probe the local dynamics of magnetic fields, thereby ruling out any long-range order. This conclusion is further supported by

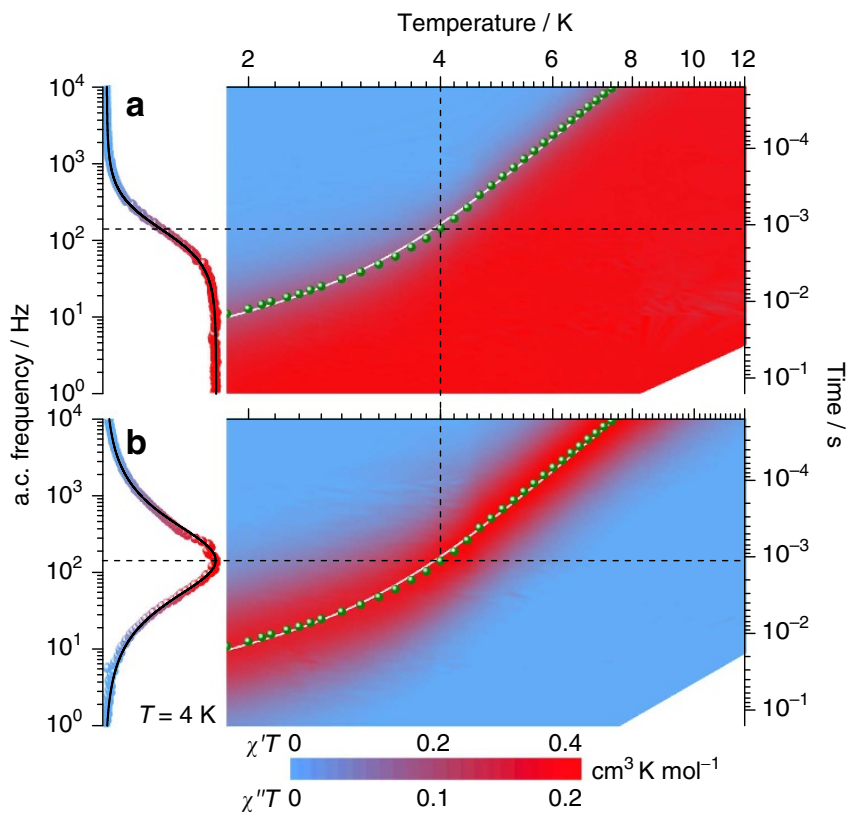

Figure 4 | Magnetization dynamics. Two-dimensional frequency/ temperature maps of the real $\left(\chi^{\prime} T, \mathbf{a}\right)$ and imaginary $\left(\chi^{\prime \prime} T, \mathbf{b}\right)$ components of the a.c. susceptibility-temperature product for a polycrystalline sample of $\mathbf{2}$ obtained under $\mu_{0} H=75 \mathrm{mT}$. Left part: representative $4-\mathrm{K}$ data of the $\chi^{\prime} T$ and $\chi^{\prime \prime} T$ frequency dependence with the solid black line being the best fit to the generalized Debye model ${ }^{27}$, that was used for each temperature to determine the relaxation time $(\tau)$ shown as green dots on the contour plots. The best fit of $\tau^{-1}$ versus $T$ is shown as solid white lines (see text). Dashed lines are guides for the eyes.

magnetization measurements on an oriented single crystal of $\mathbf{1}$ and 2 below $2 \mathrm{~K}$ (Supplementary Figs 27-30). The magnetic susceptibility $(\chi=\mathrm{d} M / \mathrm{d} H)$ estimated from these experiments in the zero-field limit follows a Curie-Weiss law, $\chi=C /(T-\theta)$, down to lowest accessible temperature of $0.03 \mathrm{~K}$, confirming the absence of magnetic order (Supplementary Fig. 31). For 2, the magnetization reaches saturation faster when the d.c. field is applied perpendicular to the $C_{4}$ axis rather than along this axis (Supplementary Figs 29 and 30), confirming the easy-plane magnetic anisotropy in agreement with the extracted $g$-factors (vide supra). The temperature and magnetic field sweep rate dependence of the magnetization revealed the existence of a weak hysteretic behaviour. At the lowest temperatures $(<0.4 \mathrm{~K})$ and in agreement with the a.c. susceptibility data (Fig. 4), butterfly-shaped $M$ versus $\mu_{0} H$ hysteresis loops for both 1 and 2 (Supplementary Figs 27-30) confirm the presence of phononbottlenecked direct processes that dominate the magnetization relaxation $^{29}$.

\section{Discussion}

The principle of dimensional reduction in solid-state structures is based on the formation of a derived compound, $\mathrm{A}_{n a} \mathrm{MX}_{x+n}$, from a parent $\mathrm{MX}_{x}$ precursor and an $\mathrm{A}_{a} \mathrm{X}$ salt, thereby forcing termination of $\mathrm{M}-\mathrm{X}-\mathrm{M}$ polymerization ${ }^{30}$. If $\mathrm{A}$ is voluminous, for instance an organic cation, child compounds with structurally and magnetically isolated molecular units can be formed. For many oxides, the ultimate dimensional reduction to molecular $\left\{\mathrm{MO}_{x}\right\}^{y-}$ is impeded by the progressive development of large localized negative charges (for example, for $\left\{\operatorname{IrO}_{6}\right\}^{8-}$ ). Thus, truly single-metal ion analogues of most oxides are difficult, if not impossible, to isolate. In the case of octahedral species, no example has been reported so far. As discussed herein for $\mathrm{Ir}^{\mathrm{IV}}$, the exchange of oxide with a less negatively charged fluoride results 
in dimensional reduction of the iridate as compared with the ternary iridium oxide parents. Although their local structures are obviously comparable, the electronic resemblance between these compounds should be discussed here. The branching ratios extracted from XAS (0.85; Table 1) are identical for $\mathbf{1}$ and $\mathbf{2}$, and very close to the values found for oxido-iridates, for example, 0.87 for $\mathrm{Sr}_{2} \mathrm{IrO}_{4}$ and 0.85 for $\mathrm{Y}_{2} \mathrm{Ir}_{2} \mathrm{O}_{7}$ (refs 31,32). In addition, the $L_{2} / L_{3}$ XMCD intensity ratio of $4.7 \%$ for 2 is almost identical to the value determined for $\mathrm{Sr}_{2} \mathrm{IrO}_{4}(\sim 5 \%)$ (ref. 33). This striking agreement demonstrates the resemblance of the electronic structure of the $\left\{\mathrm{IrO}_{6}\right\}^{8-}$ moieties in oxido-iridates and the molecular $\left[\operatorname{IrF}_{6}\right]^{2-}$ unit. A $M_{\text {orbital }} / M_{\text {spin }}=\left\langle L_{z}\right\rangle / 2\left\langle S_{z}\right\rangle$ ratio of $\sim 2.5$ in $\mathrm{Sr}_{2} \mathrm{IrO}_{4}$ was recently obtained by non-resonant magnetic $\mathrm{X}$-ray scattering that circumvents the need for the estimation of $\left\langle T_{z}\right\rangle$ (ref. 34). For comparison, $M_{\text {orbital }} / M_{\text {spin }}$ ratios of 3.3, 3.3 and 2.1 are obtained for $\mathbf{1}, \mathbf{2}$ and $\mathbf{3}$, respectively. The slightly larger ratio for $\mathbf{1}$ and 2 as compared with $\mathrm{Sr}_{2} \mathrm{IrO}_{4}$, and $\mathbf{3}$ is attributed to the weaker covalency of the $\mathrm{Ir}-\mathrm{X}$ bond for $\mathrm{X}=\mathrm{F}$ than for $\mathrm{X}=\mathrm{O}, \mathrm{Cl}$, as reflected in the nephelauxetic series ${ }^{35}$. It is worth emphasizing that the fluoride ion is the least nephelauxetic of all known ligands, and thus the $\left[\operatorname{IrF}_{6}\right]^{2-}$ moiety has the closest proximity to a perfectly ionic iridate. As a less-pronounced covalency would induce a larger $M_{\text {orbital }} / M_{\text {spin }}$ ratio ${ }^{36}$, a larger orbital magnetic moment is found in $\left[\mathrm{IrF}_{6}\right]^{2-}$ over $\left\{\mathrm{IrO}_{6}\right\}^{8-}$ and $\left[\mathrm{IrCl}_{6}\right]^{2-}$, as expected.

In oxido-iridates, the purity of the $J_{\text {eff }}=1 / 2$ state depends crucially on the structural deviation from the octahedral symmetry, rendering oxido-iridate systems with weakly distorted $\left\{\mathrm{IrO}_{6}\right\}^{8-}$ octahedra highly interesting ${ }^{37}$. Remarkably, the $\left[\mathrm{IrF}_{6}\right]^{2-}$ moiety in 2 is structurally closer to cubic than any reported oxido-iridate system. Therefore fluorido-iridates are promising materials for stabilizing an ideal $J_{\text {eff }}=1 / 2$ Mott ground state as was already concluded from the experimentally determined values of $M_{\text {orbital }}$ and $M_{\text {spin. }}$. Paramagnetic relaxation in oxido-iridates has been reported for diamagnetically doped honeycomb systems where the imperfect stoichiometry resulted in the blocking of the magnetization at low temperature ${ }^{38,39}$. Our low-temperature experiments reveal that the slow dynamics of the magnetization is indeed intrinsic to the $\mathrm{Ir}^{\mathrm{IV}}$ centre, that might have implications for quantum magnetism.

Herein, we have established that a molecular $\left[\mathrm{IrF}_{6}\right]^{2-}$ species and the $\left\{\mathrm{IrO}_{6}\right\}^{8-}$ unit, present in all oxido-iridates, possess the same electronic $J_{\text {eff }}=1 / 2$ ground state. The dominating role of the SO coupling on the peculiar magnetism intrinsic to $\mathrm{Ir}^{\mathrm{IV}}$ was elucidated by studying spatially isolated, magnetically decoupled, $\left[\mathrm{IrF}_{6}\right]^{2-}$ ions in solids. Whereas strongly perturbed by longrange magnetic interactions in iridates, the $5 \mathrm{~d}$ spin and orbital moments, as well as their local anisotropy and dynamics have been quantified. These experimental results provide a solid basis for building a realistic model to describe the unusual physics discovered in oxido-iridates. Moreover, as illustrated by the one-dimensional $\mathrm{Zn}(\mathrm{viz})_{4}\left[\mathrm{IrF}_{6}\right]$ system (2), the $\left[\mathrm{IrF}_{6}\right]^{2}-$ species appears to be a robust building-block for the construction of molecule-based architectures. It offers an unprecedented synthetic strategy using the versatile tools of soft chemistry to engineer new materials with exotic properties anticipated to be exclusively reserved for oxido-iridates.

\section{Methods}

Synthetic methods. $\mathrm{Na}_{2}\left[\mathrm{IrCl}_{6}\right] \cdot 6 \mathrm{H}_{2} \mathrm{O}$ was heated to $150^{\circ} \mathrm{C}$ in vacuo for $6 \mathrm{~h}$, cooled to room temperature (exp. (calc. for $\mathrm{Na}_{2}\left[\mathrm{IrCl}_{6}\right]$ ) weight loss $=19 \%(19 \%)$ ) and subsequently heated to $200^{\circ} \mathrm{C}$ in a flow reactor under $\mathrm{F}_{2}$ gas $(10 \%$ diluted in Ar) and kept for $6 \mathrm{~h}$. The last step was repeated to improve the crystallinity of the white product (exp. (calc. for $\mathrm{Na}_{2}\left[\operatorname{IrF}_{6}\right]$ ) weight loss $=19 \%(22 \%)$ ) that was identified as $\mathrm{Na}_{2}\left[\mathrm{IrF}_{6}\right]$ by powder X-ray diffraction (Supplementary Fig. 32). A solution of $\mathrm{Na}_{2}\left[\mathrm{IrF}_{6}\right](68 \mathrm{mg}, 0.19 \mathrm{mmol})$ in water $(1 \mathrm{ml})$ was added a solution of $\mathrm{PPh}_{4} \mathrm{Cl}(200 \mathrm{mg}, 0.53 \mathrm{mmol})$ in water $(3 \mathrm{ml})$. The resulting solution was left undisturbed for $1 \mathrm{~h}$ to produce crystalline 1 (114 mg, 59\%). Anal. calcd. (found) for $\mathrm{C}_{48} \mathrm{H}_{44} \mathrm{~F}_{6} \mathrm{IrP}_{2} \mathrm{O}_{2}$ : C: $56.46 \%$ (56.58\%), H: $4.34 \%$ (4.19\%). 1 can reversibly be oxidized to $\left[\mathrm{IrF}_{6}\right]^{-}\left(E_{1 / 2}=+0.8 \mathrm{~V}\right.$ versus $\mathrm{Fc}^{+} / \mathrm{Fc}$; Supplementary Fig. 33$)$. The addition of 1-vinylimidazole $(0.80 \mathrm{~g}, 8.5 \mathrm{mmol})$ to a methanol solution $(40 \mathrm{ml})$ of 1 $(100 \mathrm{mg}, 0.098 \mathrm{mmol})$ and $\mathrm{Zn}\left(\mathrm{NO}_{3}\right)_{2} \cdot 6 \mathrm{H}_{2} \mathrm{O}(100 \mathrm{mg}, 0.34 \mathrm{mmol})$ afforded crystals of 2 after 1 day. Yield: $60 \mathrm{mg}(82 \%)$. Anal. calcd. (found) for $\mathrm{C}_{20} \mathrm{H}_{24} \mathrm{~F}_{6} \mathrm{IrN}_{8} \mathrm{Zn}$ : C: $32.11 \%$ (32.36\%), H: 3.23\% (3.07\%), N: 14.98\% (14.67\%). Complex 3 was obtained from $\left(\mathrm{NH}_{4}\right)_{2}\left[\mathrm{IrCl}_{6}\right](203 \mathrm{mg}, 0.460 \mathrm{mmol})$ that was first dissolved in aqueous $\mathrm{HCl}$ $(0.1 \mathrm{M}, 100 \mathrm{ml})$. The solution was heated to $50^{\circ} \mathrm{C}$ and filtered hot. To this solution, a solution of $\mathrm{PPh}_{4} \mathrm{Cl}(360 \mathrm{mg}, 0.963 \mathrm{mmol})$ in aqueous $\mathrm{HCl}(0.1 \mathrm{M}, 70 \mathrm{ml})$ was added slowly, inducing an immediate precipitation of orange-brown crystals. On complete addition, the reaction mixture was cooled to room temperature and stirred for $30 \mathrm{~min}$ leaving only a slightly coloured solution. The product was filtered off and washed three times with water and dried in a dynamic vacuum. Yield: $453 \mathrm{mg}$ (91\%). Anal. calcd. (found) for $\mathrm{C}_{48} \mathrm{H}_{40} \mathrm{Cl}_{6} \mathrm{IrP}_{2}$ : C: $53.20 \%$ (53.24\%), $\mathrm{H}$ : $3.72 \%(3.67 \%)$

Crystallography. Powder X-ray diffraction patterns of $\mathrm{Na}_{2}\left[\mathrm{IrF}_{6}\right]$ were obtained on samples mounted under nitrogen in an air-tight aluminium alloy cell and collected on a PANanalytical Bragg-Brentano $\theta-2 \theta$ geometry diffractometer $(\mathrm{Cu} \mathrm{K} \alpha$ radiation) equipped with a secondary monochromator over an angular $2 \theta$ range of $5-80^{\circ}$. Rietveld refinement of the data was performed in the TOPAS (version 4) program ${ }^{40}$. Atomic positions were allowed to refine freely without restraints, while thermal parameters for all atoms were fixed. A suitable fit $\left(R_{\mathrm{wp}}=13.46 \%\right)$ was obtained using a structural model similar to that of $\mathrm{Na}_{2}\left[\mathrm{GeF}_{6}\right]$ (ref. 41), with an expanded unit cell (space group P321, $a=b=9.32858$ (24) $\AA, c=5.13417$ (19) , $\alpha=\beta=90^{\circ}, \gamma=120^{\circ}$; Supplementary Fig. 32). Single-crystal X-ray diffraction studies on 1-3 (Supplementary Table 1) were performed at 122(1) K on a Bruker D8 VENTURE diffractometer equipped with Mo K $\alpha$ high-brilliance $\mathrm{I} \mu \mathrm{S}$ radiation $(\lambda=0.71073 \AA)$, a multilayer X-ray mirror, a PHOTON 100 CMOS detector, and an Oxford Cryosystems low-temperature device. The instrument was controlled with the APEX2 software package. The structures were solved in Olex2 using the olex2.solve structure solution program (Charge Flipping) and refined using the olex2.refine program ${ }^{42}$. All non-hydrogen atoms were refined anisotropically and hydrogen atoms were placed at calculated positions and refined as riding atoms with isotropic displacement parameters.

Magnetometry. The magnetic measurements were carried out between 1.8 and $280 \mathrm{~K}$ with applied d.c. fields ranging from -9 to $+9 \mathrm{~T}$, with the use of a MPMS-XL Quantum Design SQUID magnetometer and a PPMS-9 Quantum Design susceptometer. Measurements were performed on polycrystalline samples sealed in polyethylene bags (typically $3 \times 0.5 \times 0.02 \mathrm{~cm} ; 15-30 \mathrm{mg}$ ) and immobilized in mineral oil. a.c. susceptibility measurements were performed with an oscillating field of 1-6 Oe with a frequency from 1 to $10,000 \mathrm{~Hz}$. Before the experiments, the field-dependent magnetization was measured at $100 \mathrm{~K}$ to confirm the absence of any bulk ferromagnetic impurities. The magnetic data were corrected for the diamagnetic contributions from the sample, sample holder and mineral oil. Micro-SQUID magnetic measurements on single crystals were carried out in the field range from -1.4 to $+1.4 \mathrm{~T}$ and in the $5.0-0.03 \mathrm{~K}$ temperature range The field was applied in any direction of the micro-SQUID plane with precision $>0.1^{\circ}$ by separately driving three orthogonal coils ${ }^{43}$.

X-ray spectroscopy. Polarization-dependent XAS spectra at the $\operatorname{Ir} L_{2}$ and $L_{3}$ edges were acquired at the ID12 beam line at the European Synchrotron Radiation Facility (ESRF), Grenoble, France. A helical undulator of the APPLE-II type allowed the circular polarization of incoming X-rays to be changed for each spectrum. The spectra were obtained by using the total fluorescence yield detection mode in magnetic fields up to $\pm 17 \mathrm{~T}$. Transmission detection was additionally used for $\mathbf{2}$ to check the validity of the self-absorption correction used for all samples, which took into account the chemical composition, the geometry of the experiment and the solid angle of the X-ray fluorescence detector. XMCD spectra were obtained as the difference of consecutive XAS spectra obtained with opposite photon helicities. In addition, the XMCD spectra were systematically recorded in both field directions to ensure the absence of experimental artefacts. The isotropic spectra were obtained as the sum of spectra with right and left circularly polarized $\mathrm{X}$-rays and were normalized between zero before the absorption edge and one above the edge. The position of the step function describing transitions into the continuum was defined by the following procedure. The maximum of the $L_{3}$ white line was assigned to zero energy and the $L_{2}$ edge spectrum was shifted in energy to have the first EXAFS oscillations perfectly overlapping for the two spectra. The step function was placed by visual comparison of the shifted spectra. It was systematically checked that shifting the energy of the inflection point of the step function around the chosen position $( \pm 2 \mathrm{eV})$ was not significantly altering the white line integrals. A step function broadening of $6 \mathrm{eV}$ was used, but this parameter does not affect the integrated values of the white line intensities. The white line integral at the $L_{2}$ edge was divided by 2.15 reflecting factors of (i) 2 from the occupation ratios of $2 \mathrm{p}_{3 / 2}$ and $2 \mathrm{p}_{1 / 2}$ core states and (ii) 0.15 from the fact that the sum rules are applied to the line intensities $I(\hbar \omega)$ (where $\hbar \omega$ is the photon energy) and not to the absorption cross sections, $\mu(\hbar \omega)$, which are related by $I(\hbar \omega)=1 /\left(4 \pi^{2} \alpha \hbar \omega\right) \times$ $\mu(\hbar \omega)$ (binding energies for $L_{3}$ and $L_{2}$ edges are 11.225 and $12.835 \mathrm{keV}$, 
Table 2 | Hyperfine and superhyperfine coupling parameters for $\left[\operatorname{IrF}_{6}\right]^{2}-$ doped in $\mathrm{Zn}\left(\mathrm{viz}_{4}{ }_{4}\left[\mathrm{ZrF}_{6}\right]\right.$ ( 1\% Ir).

\begin{tabular}{|c|c|c|c|c|c|c|c|c|}
\hline$A_{z}\left({ }^{191} I r\right)$ & $A_{z}\left({ }^{193} I r\right)$ & $A_{x y}\left({ }^{191} I r\right)$ & $A_{x y}\left({ }^{193} \mathrm{Ir}\right)$ & $A_{z}^{Z}$ & $A_{x y}^{Z}$ & $A_{x y}^{X Y}$ & $A_{y x}^{X Y}$ & $A_{z}^{X Y}$ \\
\hline
\end{tabular}

respectively). The same normalization procedure was applied to the corresponding $\mathrm{XMCD}$ integrals. The precise sample temperature of $2.6-2.9 \mathrm{~K}$ and the absolute magnitude of the magnetic moment at $\mu_{0} H= \pm 17 \mathrm{~T}$ was determined by scaling the field and temperature dependent XMCD signal intensity to coincide with the $M$ versus $\mu_{0} H T^{-1}$ master curve obtained from bulk magnetometry. The normalized spectra were analysed using the magneto-optical sum rules given by equation 2 for $L_{2}$ and $L_{3}$ absorption edges ${ }^{22}$ :

$$
\begin{aligned}
& \left\langle L_{z}\right\rangle=\frac{2\left\langle n_{\mathrm{h}}\right\rangle}{3} \times \frac{I_{L_{3}}^{\mathrm{XMCD}}+I_{L_{2}}^{\mathrm{XMCD}}}{I_{L_{3}}^{\mathrm{XAS}}+I_{L_{2}}^{\mathrm{XAS}}} \\
& \left\langle S_{\text {eff }}\right\rangle=\left\langle S_{z}\right\rangle+\frac{7}{2}\left\langle T_{z}\right\rangle=\frac{\left\langle n_{\mathrm{h}}\right\rangle}{2} \times \frac{I_{L_{3}}^{\mathrm{XMCD}}-2 \times I_{L_{2}}^{\mathrm{XMCD}}}{I_{L_{3}}^{\mathrm{XAS}}+I_{L_{2}}^{\mathrm{XAS}}}
\end{aligned}
$$

Ligand field and $\boldsymbol{a} \boldsymbol{b}$ initio calculations. The AOM calculations were performed using the Ligfield programme on the full $\mathrm{d}^{5}$ configuration ${ }^{44,45}$. All matrices were constructed in the weak-field basis and subsequently transformed to the eigen-basis corresponding to the parameter vectors given below. The octahedral component of the ligand field, $\Delta_{O}=27,000 \mathrm{~cm}^{-1}$, would agree with the AOM parameter $e_{\sigma}=9,000 \mathrm{~cm}^{-1}$ in the cubic model. In the more realistic model, a $e_{\sigma} / e_{\pi}$ ratio of 5 , and a distance dependence of $e_{\sigma} \propto d_{\mathrm{Ir}-\mathrm{F}}^{-5}$ and $e_{\pi} \propto d_{\mathrm{Ir}-\mathrm{F}}{ }^{-7}$ (where $d_{\mathrm{Ir}-\mathrm{F}}$ is the Ir-F bond length) was assumed (yielding $e_{\sigma}^{z}=11,904 \mathrm{~cm}^{-1}, e_{\sigma}^{x y}=12,457 \mathrm{~cm}^{-1}$, $e_{\pi}^{z}=2,356 \mathrm{~cm}^{-1}$ and $\left.e_{\pi}^{x y}=2,507 \mathrm{~cm}^{-1}\right)$. All ab initio calculations were performed with the ORCA 3.0 programme package ${ }^{46}$ using ZORA $^{47,48}$ for relativistic corrections and the def2-TZVP basis set for the $\left[\mathrm{IrX}_{6}\right]^{2-}$ moieties and the def2-SVP basis set for the 1-vinylimidazole ligands in calculations when requested in the model. State-averaged CASSCF calculations were performed on experimental geometries using Kohn-Sham starting orbitals obtained with the TPSSh functional. Active spaces of CAS $(5,3)$ (Ir t $t_{2 g}$ orbitals-3 doublet CSFs) and CAS(5,5) (Ir $d$ orbitals-12 doublet configuration state functions (CSFs), 6 quartet CSFs, 1 sextet $\mathrm{CSF}$ ) were used and the SO interaction taken into account through a mean field approximation (Supplementary Table 2$)^{49}$.

EPR spectroscopy. The spectra were acquired at $T=5 \mathrm{~K}$ on a Bruker Elexsys E500 spectrometer equipped with a Bruker ER 4116 DM dual mode cavity, an EIP 538B frequency counter and an ER035M NMR Gauss-meter, and with a microwave radiation frequency of $9.634 \mathrm{GHz}$. The spectra were simulated using home-written software considering an effective electronic $J_{\text {eff }}$ of $1 / 2$ and taking into account the natural isotopic abundance of ${ }^{191} \operatorname{Ir}(37.3 \%)$ and ${ }^{193} \operatorname{Ir}(62.3 \%)$, which have both a nuclear $I_{\mathrm{Ir}}=3 / 2 \mathrm{spin}$, as well as the ${ }^{19} \mathrm{~F}$ nuclear spin $\left(I_{\mathrm{F}}=1 / 2,100 \%\right)$. The spectra were computed using the following spin Hamiltonian for each iridium, ${ }^{191} \mathrm{Ir}$ and ${ }^{193} \mathrm{Ir}$, isotope situated in a tetragonally distorted octahedral environment:

$$
\begin{aligned}
\hat{H}= & g_{z} \mu_{\mathrm{B}} \mu_{0} \hat{S}_{z} H_{z}+g_{x y} \mu_{\mathrm{B}} \mu_{0}\left(\hat{S}_{x} H_{x}+\hat{S}_{y} H_{y}\right) \\
& +A_{z}^{\mathrm{Ir}} \hat{S}_{z} \hat{I}_{z}+A_{x y}^{\mathrm{Ir}}\left(\hat{S}_{x} \hat{I}_{x}+\hat{S}_{y} \hat{I}_{y}\right) \\
& +A_{z}^{\mathrm{Z}} \hat{S}_{z}\left(\hat{I}_{5, z}+\hat{I}_{6, z}\right)+A_{x y}^{\mathrm{Z}}\left[\hat{S}_{x}\left(\hat{I}_{5, x}+\hat{I}_{6, x}\right)+\hat{S}_{y}\left(\hat{I}_{5, y}+\hat{I}_{6, y}\right)\right] \\
& +A_{x y}^{\mathrm{XY}}\left[\hat{S}_{x}\left(\hat{I}_{1, x}+\hat{I}_{2, x}\right)+\hat{S}_{y}\left(\hat{I}_{3, y}+\hat{I}_{4, y}\right)\right]+A_{z}^{\mathrm{XY}} \hat{S}_{z}\left(\hat{I}_{1, z}+\hat{I}_{2, z}+\hat{I}_{3, z}+\hat{I}_{4, z}\right) \\
& +A_{y x}^{\mathrm{XY}}\left[\hat{S}_{x}\left(\hat{I}_{3, x}+\hat{I}_{4, x}\right)+\hat{S}_{y}\left(\hat{I}_{1, y}+\hat{I}_{2, y}\right)\right]
\end{aligned}
$$

Here, $\hat{S}$ operators refer to the effective spin of $J_{\text {eff }}=1 / 2$ pertinent to the electronic ground state. $\hat{I}_{x}, \hat{I}_{y}$ and $\hat{I}_{z}$ operators refer to the nuclear spin of the iridium nucleus and $\hat{I}_{n, x}, \hat{I}_{n, y}, \hat{I}_{n, z}(n=1,2,3,4,5$ or 6$)$ refer to the nuclear spin of the fluoride ligands. The fluoride ligands on the positive and negative $\mathrm{X}$-axis are designated with subscripts ' 1 ' and ' 2 ', respectively. Similarly, subscripts ' 3 ' and ' 4 ', and subscripts ' 5 ' and ' 6 ', designate the fluoride ligands on the $\mathrm{Y}$ - and $\mathrm{Z}$-axis, respectively. The extracted parameter values are given in Table 2. Remarkably, the estimated hyperfine interactions appear to be almost isotropic, in contrast to the strongly anisotropic superhyperfine interactions to the fluoride ions, which exhibit averaged values on the same order of magnitude as the hyperfine ones. Performing the same experiment on a concentrated crystal of $\mathbf{2}$ gave virtually identical $g$-factors, but the hyperfine interactions could not be resolved.

Muon-spin relaxation. The $\mu^{+}$SR measurements were performed on the Dolly spectrometer at the Paul Scherrer Institut (Villigen, Switzerland). Fully spin-polarized muons were implanted into the sample and acted as a local probe of the internal magnetic fields. The time evolution of the polarization, which was monitored via the anisotropic $\beta$-decay of the implanted muons (lifetime $2.2 \mu \mathrm{s}$ ), is determined by the temporal and spatial properties of the local magnetic field ${ }^{50}$.
For example, fluctuating magnetic moments in the vicinity of a muon would produce a fluctuating dipolar magnetic field, and therefore, a polarization relaxation from its initial value to zero. The time scale of the relaxation can be extracted from the field dependence of the muon spin-lattice relaxation, $\lambda$, considering the following equation ${ }^{51}$,

$$
\lambda=\frac{\Delta^{2} \tau}{1+\gamma^{2} \tau^{2}\left(\mu_{0} H\right)^{2}}
$$

where, $\Delta$ is the magnitude of the local magnetic field, $\gamma$ is the gyromagnetic ratio of the muon, $\mu_{0} H$ is the applied magnetic field and $\tau$ is the fluctuation time scale. The equation above assumes that $\Delta$ and $\tau$ are field-independent. From the fit of $\lambda$ as a function of $\mu_{0} H$ (Supplementary Fig. 26) $\tau \sim 0.016 \mu$ s is obtained. The small value of $\tau$ compared with that obtained from a.c. susceptibility measurements is due to the fact that $\mu \mathrm{SR}$ reflects the integrated contribution over $q$-space as a local, point-like probe, while a.c. susceptibility measures the response for $q=0$ (ref. 52).

Data availability. The X-ray crystallographic coordinates for structures reported in this Article have been deposited at the Cambridge Crystallographic Data Centre (CCDC), under deposition number CCDC 1431835-1431837. These data can be obtained free of charge from The Cambridge Crystallographic Data Centre via www.ccdc.cam.ac.uk/data_request/cif. All other relevant data are available from the authors on request.

\section{References}

1. Kim, B. J. et al. Phase-sensitive observation of a spin-orbital Mott state in $\mathrm{Sr}_{2} \mathrm{IrO}_{4}$. Science 323, 1329-1332 (2009).

2. Kim, B. J. et al. Novel $J_{\text {eff }}=1 / 2$ Mott state induced by relativistic spin-orbit coupling in $\mathrm{Sr}_{2} \mathrm{IrO}_{4}$. Phys. Rev. Lett. 101, 076402 (2008).

3. Pesin, D. \& Balents, L. Mott physics and band topology in materials with strong spin-orbit interaction. Nat. Phys. 6, 376-381 (2010).

4. Chen, Y., Lu, Y.-M. \& Kee, H.-Y. Topological crystalline metal in orthorhombic perovskite iridates. Nat. Commun. 6, 6593 (2015).

5. Kondo, T. et al. Quadratic Fermi node in a $3 \mathrm{D}$ strongly correlated semimetal. Nat. Commun. 6, 10042 (2015).

6. Kim, Y. K. et al. Fermi arcs in a doped pseudospin-1/2 Heisenberg antiferromagnet. Science 345, 187-190 (2014).

7. Kim, Y. K., Sung, N. H. \& Kim, B. J. Observation of a $d$-wave gap in electron-doped $\mathrm{Sr}_{2} \mathrm{IrO}_{4}$. Nat. Phys. 12, 37-41 (2016).

8. Zhao, L. et al. Evidence of an odd-parity hidden order in a spin-orbit coupled correlated iridate. Nat. Phys. 12, 32-37 (2016).

9. Machida, Y., Nakatsuji, S., Onoda, S., Tayama, T. \& Sakakibara, T. Time-reversal symmetry breaking and spontaneous Hall effect without magnetic dipole order. Nature 463, 210-213 (2010).

10. Modic, K. A. et al. Realization of a three-dimensional spin-anisotropic harmonic honeycomb iridate. Nat. Commun. 5, 4203 (2014).

11. Nishimoto, S. et al. Strongly frustrated triangular spin lattice emerging from triplet dimer formation in honeycomb $\mathrm{Li}_{2} \mathrm{IrO}_{3}$. Nat. Commun. 7, 10273 (2016).

12. Tian, Z. et al. Field-induced quantum metal-insulator transition in the pyrochlore iridate $\mathrm{Nd}_{2} \mathrm{Ir}_{2} \mathrm{O}_{7}$. Nat. Phys. 12, 134-139 (2016).

13. Ballhausen, C. J. Introduction to Ligand Field Theory (McGraw-Hill, 1962).

14. Witczak-Krempa, W., Chen, G., Kim, Y. B. \& Balents, L. Correlated quantum phenomena in the strong spin-orbit regime. Annu. Rev. Condens. Matter Phys. 5, 57-82 (2014).

15. Chun, S. H. et al. Direct evidence for dominant bond-directional interactions in a honeycomb lattice iridate $\mathrm{Na}_{2} \mathrm{IrO}_{3}$. Nat. Phys. 11, 462-466 (2015).

16. Bogdanov, N. A. et al. Orbital reconstruction in nonpolar tetravalent transitionmetal oxide layers. Nat. Commun. 6, 7306 (2015)

17. Birol, T. \& Haule, K. $J_{\text {eff }}=1 / 2$ Mott-insulating state in Rh and Ir fluorides. Phys. Rev. Lett. 114, 096403 (2015)

18. Hepworth, M. A. et al. Complex fluorides of iridium and osmium. J. Chem. Soc. 4269-4275 (1954).

19. Bartlett, N. \& Tressaud, A. Sur un nouveau type structural de tétrafluorures d'éléments de transition: synthèse, étude cristallographique et magnétique de $\mathrm{IrF}_{4}$. C. R. Acad. Sci. 278, 1501-1505 (1974).

20. Rao, P. R., Tressaud, A. \& Bartlett, N. The tetrafluorides of iridium, rhodium and palladium. J. Inorg. Nucl. Chem. Suppl. 1 28, 23-28 (1976).

21. van der Laan, G. \& Thole, B. T. Local probe for spin-orbit interaction. Phys. Rev. Lett. 60, 1977-1980 (1988). 
22. Thole, B. T., Carra, P. \& van der Laan, G. X-ray circular dichroism as a probe of orbital magnetism. Phys. Rev. Lett. 68, 1943-1946 (1992).

23. Carra, P., Thole, B. T., Altarelli, M. \& Wang, X. X-ray circular dichroisms and local magnetic fields. Phys. Rev. Lett. 70, 694-697 (1993).

24. Laguna-Marco, M. A. et al. Orbital magnetism and spin-orbit effects in the electronic structure of $\mathrm{BaIrO}_{3}$. Phys. Rev. Lett. 105, 216407 (2010).

25. Jørgensen, C. K., Pappalardo, R. \& Schmidtke, H.-H. Do the 'Ligand' field parameters in lanthanides represent weak covalent bonding? J. Chem. Phys. 39, 1422-1430 (1963).

26. Allen, G. C., Al-Mobarak, R., El-Sharkawy, G. A. M. \& Warren, K. D. The electronic spectra of the hexahalo anions of osmium(IV) and iridium(IV). Inorg. Chem. 11, 787-796 (1972).

27. Cole, K. S. \& Cole, R. H. Dispersion and absorption in dielectrics. J. Chem. Phys. 9, 341-351 (1941).

28. Orbach, R. Spin-lattice relaxation in rare-earth salts. Proc. R. Soc. London, Ser. A 264, 458-484 (1961).

29. Chiorescu, I., Wernsdorfer, W., Müller, A., Miyashita, S. \& Barbara, B. Adiabatic Landau-Zener-Stückelberg transition with or without dissipation in the low-spin molecular system $\mathrm{V}_{15}$. Phys. Rev. B 67, 020402 (2003).

30. Tulsky, E. G. \& Long, J. R. Dimensional reduction: a practical formalism for manipulating solid structures. Chem. Mater. 13, 1149-1166 (2001).

31. Clancy, J. P. et al. Spin-orbit coupling in iridium-based $5 d$ compounds probed by X-ray absorption spectroscopy. Phys. Rev. B 86, 195131 (2012).

32. Katukuri, V. M. et al. Electronic structure of low-dimensional $4 \mathrm{~d}^{5}$ oxides: interplay of ligand distortions, overall lattice anisotropy, and spin-orbit interactions. Inorg. Chem. 53, 4833-4839 (2014).

33. Haskel, D. et al. Pressure tuning of the spin-orbit coupled ground state in $\mathrm{Sr}_{2} \mathrm{IrO}_{4}$. Phys. Rev. Lett. 109, 027204 (2012).

34. Fujiyama, S. et al. Spin and orbital contributions to magnetically ordered moments in $5 d$ layered perovskite $\mathrm{Sr}_{2} \mathrm{IrO}_{4}$. Phys. Rev. Lett. 112, 016405 (2014).

35. Tchougréeff, A. I. \& Dronskowski, R. Nephelauxetic effect revisited. Int. J. Quant. Chem. 109, 2606-2621 (2009).

36. Stevens, K. W. H. On the magnetic properties of covalent $\mathrm{XY}_{6}$ complexes. Proc. R. Soc. Lond. A 219, 542-555 (1953).

37. Calder, S. et al. $J_{\mathrm{eff}}=1 / 2$ Mott spin-orbit insulating state close to the cubic limit in $\mathrm{Ca}_{4} \mathrm{IrO}_{6}$. Phys. Rev. B 89, 081104 (2014).

38. Manni, S., Tokiwa, Y. \& Gegenwart, P. Effect of nonmagnetic dilution in the honeycomb-lattice iridates $\mathrm{Na}_{2} \mathrm{IrO}_{3}$ and $\mathrm{Li}_{2} \mathrm{IrO}_{3}$. Phys. Rev. B 89, 241102 (2014).

39. Wallace, D. C., Brown, C. M. \& McQueen, T. M. Evolution of magnetism in the $\mathrm{Na}_{3-\delta}\left(\mathrm{Na}_{1-x} \mathrm{Mg}_{x}\right) \mathrm{Ir}_{2} \mathrm{O}_{6}$ series of honeycomb iridates. J. Solid State Chem. 224, 28-35 (2015).

40. Coelho, A. A. TOPAS Academic, Version 4; Coelho Software: Brisbane, Australia (2012)

41. Averdunk, F. \& Hoppe, F. Zur synthese von einkristallen komplexser fluoride der halbmetalle auf trockenem wege: $\mathrm{Li}_{2}\left(\mathrm{GeF}_{6}\right)$ und $\mathrm{Na}_{2}\left(\mathrm{GeF}_{6}\right)$. Z. Anorg. Allg. Chem. 582, 111-120 (1990).

42. Dolomanov, O. V., Bourhis, L. J., Gildea, R. J., Howard, J. A. K. \& Puschmann, H. OLEX2: a complete structure solution, refinement and analysis program. J. Appl. Cryst. 42, 339-341 (2009).

43. Wernsdorfer, W. From micro- to nano-SQUIDs: applications to nanomagnetism. Supercond. Sci. Technol. 22, 064013 (2009).

44. Bendix, J. in Comprehensive Coordination Chemistry II, From Biology to Nanotechnology (ed. Lever, A. B. P.) Vol 2, 673-676 (Elsevier, 2003).

45. Bendix, J., Brorson, M. \& Schaffer, C. E. Accurate empirical Spin-Orbit coupling parameters $\zeta_{n d}$ for gaseous $n \mathrm{~d}^{q}$ transition metal ions-the parametrical multiplet term model. Inorg. Chem. 32, 2838-2849 (1993).

46. Neese, F. ORCA, An Ab Initio, DFT and Semiempirical electronic structure package v. 3.0.

47. van Lenthe, E., van der Avoird, A. \& Wormer, P. E. S. Density functional calculations of molecular hyperfine interactions in the zero order regular approximation for relativistic effects. J. Chem. Phys. 108, 4783-4796 (1998).

48. van Wüllen, C. Molecular density functional calculations in the regular relativistic approximation: method, application to coinage metal diatomics, hydrides, fluorides and chlorides, and comparison with first-order relativistic calculations. J. Chem. Phys. 109, 392-399 (1998).

49. Neese, F. Efficient and accurate approximations to the molecular spin-orbit coupling operator and their use in molecular g-tensor calculations. J. Chem Phys. 122, 034107 (2005).

50. Yaouanc, A. \& Dalmas de Réotier, P. Muon Spin Rotation, Relaxation, and Resonance: Applications to Condensed Matter (OUP, 2010).

51. Salman, Z. et al. Dynamics at $\mathrm{T} \rightarrow 0$ in half-integer isotropic magnetic molecules. Phys. Rev. B 65, 132403 (2002).

52. Uemura, Y. Interplays of $\mu \mathrm{SR}$, susceptibility and neutron studies of dilute-alloy spin glasses. J. Hyperfine Interact. 18, 447-452 (1984).

\section{Acknowledgements}

We thank E. Lebraud and P. Voisin for technical and experimental assistance on powder $\mathrm{X}$-ray diffraction and on XAS/XMCD experiments, respectively. The $\mu^{+} \mathrm{SR}$ and X-ray spectroscopy experiments were performed at the Paul Scherrer Institute (Swiss Muon Source $(\mathrm{S} \mu \mathrm{S})$, Villigen, Switzerland and at the European Synchrotron Radiation Facility (ESRF, Grenoble, France), respectively. Y.L. and W.W. thank the EU for financial support within the FP7 FET-Proactive project MoQuaS No610449 and the Agence Nationale de la Recherche (ANR) project MolQuSpin, ANR-13-BS10. K.S.P. and R.C. thank the Danish Research Council for Independent Research for a DFF-Sapere Aude Research Talent grant (4090-00201), the University of Bordeaux, the Région Aquitaine, the ANR, the CNRS and the GdR MCM-2: Magnétisme et Commutation Moléculaires.

\section{Author contributions}

K.S.P., J.B. and R.C. designed the research project. K.S.P., A.T., E.D. and J.B. synthesized the compounds and developed the fluorination technologies. K.S.P. and D.N.W performed the structural analysis. R.C., W.W., Y.L. and K.S.P. acquired and analysed the magnetic data. K.S.P., A.R., K.O., F.W., C.M., S.P. and R.C. acquired and analysed the X-ray spectroscopic data. H.W. and K.S.P. performed the EPR experiments and analysed the data. J.B., T.J.M., S.I.K. and S.O. performed the ligand field and quantum chemical calculations. Z.S. and K.S.P. obtained and analysed the $\mu^{+}$SR data.

\section{Additional information}

Supplementary Information accompanies this paper at http://www.nature.com/ naturecommunications

Competing financial interests: The authors declare no competing financial interests.

Reprints and permission information is available online at http://npg.nature.com/ reprintsandpermissions/

How to cite this article: Pedersen, K. S. et al. Iridates from the molecular side. Nat. Commun. 7:12195 doi: 10.1038/ncomms12195 (2016).

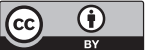

This work is licensed under a Creative Commons Attribution 4.0 International License. The images or other third party material in this article are included in the article's Creative Commons license, unless indicated otherwise in the credit line; if the material is not included under the Creative Commons license, users will need to obtain permission from the license holder to reproduce the material. To view a copy of this license, visit http://creativecommons.org/licenses/by/4.0/

(C) The Author(s) 2016 


\section{Erratum: Iridates from the molecular side}

Kasper S. Pedersen, Jesper Bendix, Alain Tressaud, Etienne Durand, Høgni Weihe, Zaher Salman, Thorbjørn J. Morsing, Daniel N. Woodruff, Yanhua Lan, Wolfgang Wernsdorfer, Corine Mathonière, Stergios Piligkos,

Sophia I. Klokishner, Serghei Ostrovsky, Katharina Ollefs, Fabrice Wilhelm, Andrei Rogalev \& Rodolphe Clérac

Nature Communications 7:12195 doi: 10.1038/ncomms12195 (2016); Published 20 Jul 2016; Updated 31 Aug 2016

The original version of this Article contained an error in which the first affiliation was incorrectly given as 'CNRS, ICMCB, UPR 9048, Pessac 33600, France'. This has now been corrected in both the PDF and HTML versions of the Article.

(c) (i) This work is licensed under a Creative Commons Attribution 4.0 International License. The images or other third party material in this article are included in the article's Creative Commons license, unless indicated otherwise in the credit line; if the material is not included under the Creative Commons license, users will need to obtain permission from the license holder to reproduce the material. To view a copy of this license, visit http://creativecommons.org/licenses/by/4.0/

(C) The Author(s) 2016 